\title{
Applications of Terrain and Sensor Data Fusion in Image Mining
}

\author{
Krzysztof Koperski, Giovanni Marchisio, Selim Aksoy, and Carsten Tusk \\ Insightful Corporation \\ 1700 Westlake Ave. N, Suite 500 \\ Seattle, WA, 98109-3044 \\ \{krisk, giovanni, saksoy, ctusk\}@insightful.com
}

\begin{abstract}
We describe usage of DEM data in the VisiMine system for data mining and statistical analysis of the collections of remotely sensed images.
\end{abstract}

\section{INTRODUCTION}

Fusion of terrain with optical and other sensor data can greatly enhance many image processing and interpretation tasks. In a broader sense we could employ observed statistical interdepencies of optical and terrain data over large areas to solve problems in automatic image classification in an entirely probabilistic framework. This would benefit our ability to predict vegetation types and land classes, model deviations in multispectral reflectance, reduce the time required for spectral signature calibration and terrain correction, and generally improve the precision of many data mining operations.

We provide a scientific workbench that integrates a high performance engine for statistical data mining (the S system, winner of the 1998 ACM award) with an extensive image processing library and a relational database management system. We employ a fast multiscale segmentation algorithm to compute and store alternative polygon decompositions of a scene based on terrain or sensor data. Topological operations such as joins and intersections may unravel where sensor and terrain feature events are spatially coincident or separate. We use this data mining platform to model multivariate and probabilistic dependencies of sensor derived attributes (like spectral classes, statistical or structural textural properties, fractal scale, shape, area and orientation of optically derived polygons) on terrain data (slope, aspect, and surface roughness). We demonstrate a number of data mining tasks, which include content based searches and clustering, multivariate analyses of terrain and optical attributes, and hierarchical classification of database contents with decision trees, where the predictor variables are terrain derived features.

\section{FeATURE EXTRACTION AND DATA StORAGE}

VisiMine project provides the infrastructure and methodology required for the analysis of satellite images. Large images are partitioned into a number of smaller, more manageable image tiles. In addition to providing faster extraction of segments, partitioning allows fetching of just the relevant tiles when retrieval of only part of the image is requested. Then those individual image tiles are processed to extract the feature vectors. There are three levels of features in the VisiMine system: pixel, region, and tile levels.

Pixel level features describe spectral and textural information about each pixel. Polygon level features describe connected groups of pixels, which are produced by the segmentation process that uses a function based on the algorithm presented in [1]. This function segments an input image into non-overlapping regions by minimizing an energy functional that trades off the similarity of regions against the length of their shared boundary. It starts by breaking the image into many small regions. The algorithm then merges into a single region the two adjoining regions that are the most alike in terms of the specified polynomial model, given the length of the border between the two regions. To achieve segmentation uniformity between the different tiles, the final value of lambda is set to be approximately the same for each image tile.

In the case of multi-band satellite images, the values of the pixels are often correlated. Therefore, a Principal Component Analysis is performed based on a large sample of pixels from all tiles, all tiles are rotated to the same axes and, for LANDSAT images, the first three components are used for the segmentation of each tile. In order to avoid oversegmentation of clouds, snow, glaciers and urban area, the values of pixels rotated to principal components are saturated to mean $+-2 \mathrm{x}$ std.dev.

The data used in the segmentation process may also include DEM and DEM derived information such as aspect and slope. This may be used for classification in order to account for terrain corrections and different types of vegetation w.r.t. the orientation of the slope in the mountainous areas.

Spatial information about region levels also can be stored in ESRI's Spatial Data Engine (SDE), together with the relevant GIS information. SDE provides open data access across local and wide area networks, and the Internet, using the TCP/IP protocol. This information can be combined with region level features such as texture, spectral properties, or DEM features. Other ESRI products, such as, ArcInfo, ArcView, and MapObjects can access the data stored in the SDE, together with additional map data. The SDE format allows additional fusion of GIS, optical, and DEM information for a variety of visualization methods and data analysis functions.

Following the segmentation process, each polygon is described by its boundary and by a number of attributes that present information about the content of the region in terms of shape, size, etc. The spectral and texture properties are based on pixel features of point within the polygon. Tile level features present spectrum and texture information about whole image tiles. 
All image features, together with the original images, are stored in a database system, and they are indexed for fast retrieval. The auxiliary raster data such as Digital Elevation Models (DEM) are also stored in the database and used for feature extraction and data analysis. The Oracle database system provides the means for fast information retrieval and network accessibility.

\section{SimiLARITY SEARCH}

VisiMine uses an SQL-like query language that enables specification of the data mining task, the features that are to be used in the mining process, and any additional constraints. The system is capable of performing similarity searches based on any combination of features. A user can look for the most similar image tiles, or for the most similar regions based on a pattern tile or a pattern region. VisiMine allows arbitrary weighting of the features. The values of the features can be adjusted to have the range $[0,1]$, they can be multiplied by a specific value, or they can remain the same.

Fig. 1 presents the results of the similarity search for snowcapped mountains using Gabor texture features. When the elevation constraints are not specified regions that have different land cover are also retrieved. To focus search the elevation constraint was added to the query as it is presented below.

\section{FIND SIMILAR TO SELECTED REGION \\ IN RELEVANCE TO A.IMAGETILEID, A.REGIONID, \\ A.C0, A.C1, A.C2, A.C3, A.C4, A.C5, A.C6, A.C7, A.C8, \\ A.C9, A.C10, A.C11, A.C12, A.C13, A.C14, A.C15, A.C16, \\ A.C17, A.C18, A.C19, A.C20, A.C21, A.C22, A.C23, A.C24 \\ FROM GEOBROWSE.LANDSAT_GABOR_25_CL_R A, GEOBROWSE.LANDSAT SEGM R B \\ WHERE A.IMAGETILEID $=\bar{B}$.IMAGETILEID AND \\ A.REGIONID = B.REGIONID AND B.DEM_AVG > 1600}

The results of this query are shown in Fig. 2.

\section{Data Mining FunCtionality}

In addition to the similarity search, the VisiMine system provides functionality for other types of analyses of remotely sensed data. The data mining power of VisiMine includes similarity searches on tile and polygon levels, clustering of tiles, classification and regression analysis, searching and classification using visual grammar, label training using Bayesian and tree models, and connection to S-PLUS with over 3000 statistical functions. The data mining queries are specified in an SQL-like language. A user may specify the features that are used in the mining task and constraints used to select data for the mining process. The graphical query constructor enables fast creation of the queries by nontechnical users.

The user has high level of flexibility in choosing the features and images used for data analysis. The graphical user interface enables presentation of the models on high and general level as well as drilling down into the details. The label training enables interactive definition of models for land cover labels.

In many cases it is very difficult to describe analytically the features of the objects that a user is interested in.

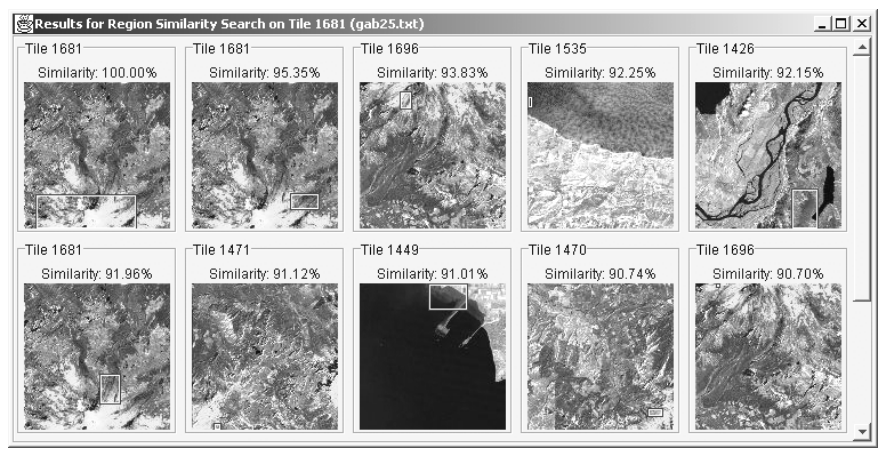

Fig. 1: Gabor 25 Clusters region similarity search

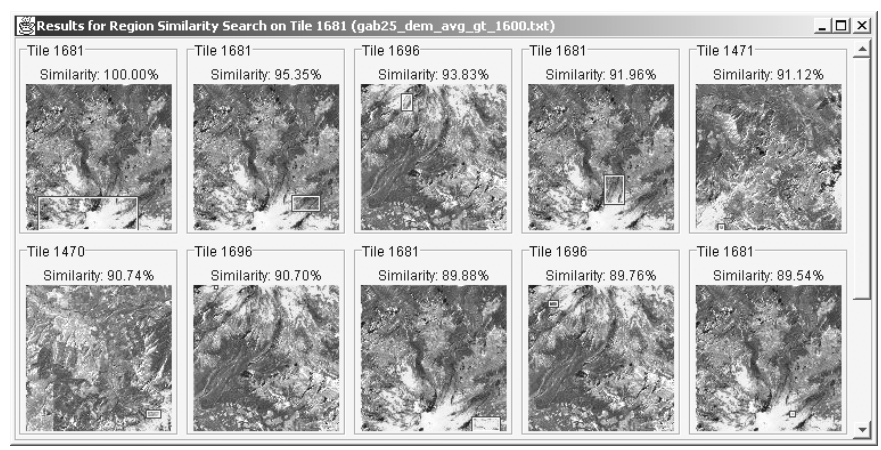

Fig. 2: Gabor 25 Clusters region similarity search with the condition DEM $A V G>1600 \mathrm{~m}$

Therefore, improvement of the quality of the description may play an important role in image analysis. A method for interactive training of land cover labels using naïve Bayesian classifiers is described in [2]. In that approach, a user can interactively train a Bayesian model to define a number of land cover classes, which can be based on textural, spectral, or DEM properties of images and terrain. The training is done based on pixel level features partitioned into a number of clusters. First, beginning with a single image tile, a user provides positive examples by selecting regions with pixels that belong to the same class, and the system then builds a model for use in identification of additional regions belonging to that class. He/she also has to provide negative examples, by selecting regions with pixels that belong to other classes. Based on this information, a model that estimates the posteriori probability of a pixel's class membership is built. The probability of pixels of the selected tile is shown on the screen. When a user judges the model to be good enough for pixels in the currently selected tile, he/she poses a query that finds either images with a high probability of belonging to the defined class, or images with a low probability (high separability from the class). Using these images, the user may choose to continue training based on other image tiles until the model is judged to be sufficiently good.

While the training is based on pixel level features, the retrieval is based on tile level features. Due to the nature of the naïve Bayesian classifier, which assumes conditional independence of the attributes, it is possible to find the average probabilities of the pixel class assignments in a tile 
based on the aggregated information about all pixels in the image tile. Despite the fact that the assumption of conditional independence is not always true, naïve Bayesian classifiers perform well in practice.

Another method for label training is based on decision tree models [3]. Because the classification process on the pixel level would be extremely expensive to compute, the decision tree models are based on the region level features. In addition to spectral properties of the regions we can perform classification based on shape properties and areas of regions, as well as on auxiliary GIS information. For example, the spectral reflectance of concrete is very similar to spectral reflectance of different types of rocks. Additional information, such as Digital Elevation Models can be used to distinguish between these two types of land cover. In a way similar to Bayesian label training, a user provides the system with positive examples by pointing to regions that belong to the trained class, and negative examples by pointing to other regions. Fig. 3 shows the results of decision tree label training that utilizes region spectral class histograms and DEM information. The examples of tidal flats are presented to the system and the regions with the highest coverage of the regions with high probability to be a tidal flat according to the model are shown. The decision tree is presented the highlighted node presents the split based on the average elevation of the region.

\section{S-PLUS CONNECTION}

Insightful's S-PLUS [3] is an interactive computing environment for graphics, data analysis, statistics, and mathematical computing. It contains a super-set of the $\mathrm{S}$ object-oriented language and system originally developed at AT\&T Bell Laboratories, and it provides an environment for high-interaction graphical analysis of multivariate data, modern statistical methods (e.g., robust and non-parametric methods), data clustering and classification, and mathematical computing. In total, S-PLUS contains over 3000 functions for scientific data analysis. VisiMine data can be accessed from within S-PLUS by using Java connectivity for images and ODBC connectivity for image and region data. In addition, VisiMine has the S-PLUS command tool (Figure 16), which provides for easy transfer of images to SPLUS, and for data processing using the S-PLUS language. The S-PLUS images can be returned to VisiMine and displayed there. The interface to S-PLUS also allows display of S-PLUS graphics. The graphics are shown in S-PLUS plot windows, and they can be created using functions typed into the execution window.

\section{CONCLUSIONS}

The combination of S-PLUS and VisiMine features creates a unique environment for interactive exploration and analysis of remotely sensed data. The rich statistical functionality of S-PLUS, together with the VisiMine User Interface and the scalability of its data mining engine, allows for easy and powerful customization of the data analysis process.

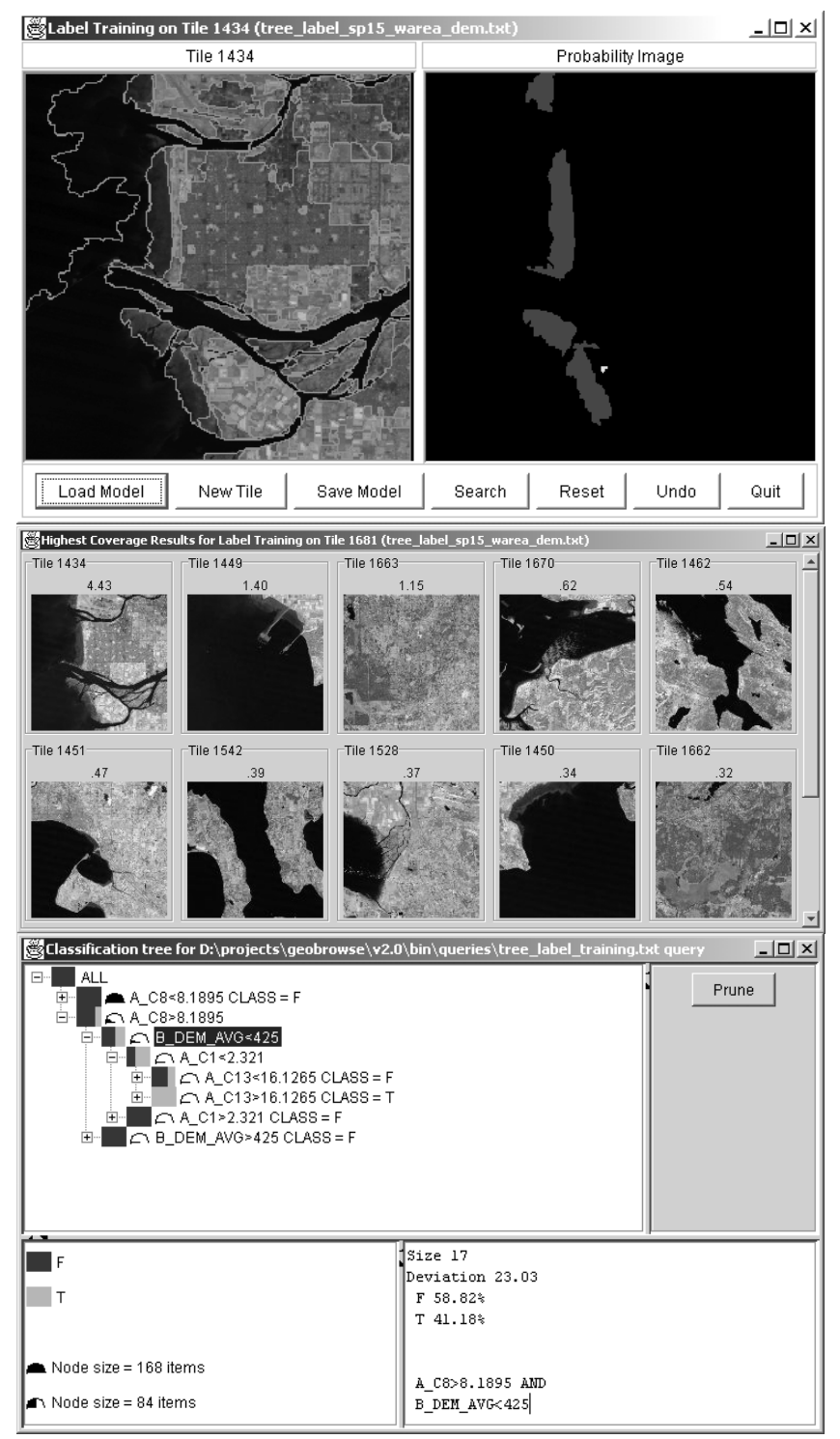

Fig. 3. Label training using region level spectral and elevation features.

\section{ACKNOWLEDGEMENTS}

Funding for the prototype comes from NASA SBIR Phase II contract NAS5-98053 and NRA2-37143 contract.

\section{REFERENCES}

[1] Koepfler, G., C. Lopez and J. M. Morel, A Multiscale Algorithm for Image Segmentation by Variational Method, SIAM Journal of Numerical Analysis, Vol. 31, pp. 282 - 299, 1994.

[2] Schröder, M., H. Rehrauer, K. Seidel, and M. Datcu. Interactive Learning and Probabilistic Retrieval in Remote Sensing Image Archives. In IEEE Tran. on Geoscience and Remote Sensing, Sep. 2000, Vol. 38, No. 5 pp. 2288 - 2298.

[3] S-PLUS 2000 Programmer's Guide, Data Analysis Products Division, Mathsoft, Seattle, WA, 1999. 